\title{
Delayed Diagnosis
}

\section{Malignant astrocytoma of the cervico-medullary junction masquerading as Guillain-Barré syndrome}

\author{
S.C. Beards, L.J. Robertson, A. Jackson ${ }^{1}$ and J. Lipman
}

Baragwanath Intensive Care Unit and Department of Anaesthesia, University of Witwatersrand, PO Box 2013, Bertsham, Soweto, South Africa, and ${ }^{1}$ Department of Neuroradiology, Manchester Royal Infirmary, Oxford Road, Manchester M13 9WL, UK

\begin{abstract}
Summary: Brainstem gliomas are rare primary brain tumours which most commonly occur in the midbrain and pons. Malignant gliomas and tumours at the cervico-medullary junction are particularly unusual. The diagnosis of tumours at this site is particularly difficult using computed tomographic (CT) scanning owing to artifacts around the base of the skull. Intrinsic tumours of the cervico-medullary junction may lead to a dissociated motor deficit and the onset of symptoms can be rapid. We describe a patient in whom an isolated ascending motor deficit in association with a raised cerebrospinal fluid protein and a normal CT scan led to an erroneous diagnosis of Guillain-Barré syndrome. The patient was treated on the intensive care unit for an 8-week period before further investigation demonstrated a malignant glioma of the cervico-medullary junction. We recommend confirmation of the diagnosis of polyradiculopathy by nerve conduction studies wherever possible.
\end{abstract}

\section{Introduction}

The typical clinical presentation of Guillain-Barré syndrome is one of areflexic flaccid and symmetrical motor paralysis associated with an elevated protein and normal cell count in the cerebrospinal fluid (CSF). Motor involvement of the cranial nerves is seen in up to $50 \%$ but sensory symptoms are usually minor. The onset of weakness usually occurs over a period of several days and often follows a prodromal infective illness. Motor symptoms may progress for up to 4 weeks, after which there is a stable period followed by a gradual and slow improvement which may be delayed for up to 2 months. ${ }^{1.2}$ We present a patient in whom the clinical progression and laboratory findings were typical of Guillain-Barré syndrome and a computed tomographic (CT) scan of the head was normal. He was given a full course of immunoglobulins and ventilated for 10 weeks before neurological deterioration led to the diagnosis of a malignant astrocytoma of the cervico-medullary junction.

Correspondence: A. Jackson, Ph.D., M.B., Ch.B., M.R.C.P., F.R.C.R., Department of Neuroradiology, Manchester Royal Infirmary, Oxford Road, Manchester M13 9WL, UK.

\section{Case report}

A 33 year old male presented with a one week history of progressive ascending weakness, headache and respiratory difficulty. His symptoms developed 3 weeks after a prodromal illness characterized by a sore throat and productive cough. On examination he was severely dyspnoeic. His heart rate was 110 beats per minute, blood pressure $150 / 80 \mathrm{mmHg}$, respiratory rate 36 per minute and temperature $38.4^{\circ} \mathrm{C}$. His chest was clear to auscultation. He had severe (Grade IV) motor weakness in both upper and lower limbs with depressed peripheral reflexes, and downgoing plantar responses. There were no cranial nerve deficits and no signs of sensory deficit despite paraesthesia in both hands and feet. The fundi were normal and there was no evidence of meningism. The chest X-ray demonstrated minor bilateral lower zone consolidation.

Arterial blood gases showed evidence of type II respiratory failure. The patient was intubated and mechanically ventilated and tranferred to the intensive care unit. An urgent CT scan was performed both with and without intravenous iodinated contrast. Images were obtained from the $\mathrm{C} 2$ vertebra to the vertex and revealed no apparent abnormality. The fourth ventricle was midline and there was no evidence of either abnormal enhancement or mass effect within the posterior fossa or upper 
spinal canal (Figure 1). Laboratory investigations demonstrated a leucocytosis of $11.3 \times 10^{9} / 1$, other routine haematological and biochemical investigations were normal. Lumbar puncture yielded clear colourless CSF, the protein was markedly elevated $(1.19 \mathrm{~g} / \mathrm{l})$ but microscopy showed no cells and CSF glucose and chloride levels were normal.

After a period of initial stabilization, the patient required continuing ventilatory support with an inspired oxygen concentration of 0.5 to maintain an acceptable $P_{\mathrm{a}} \mathrm{O}_{2}$. Neurological examination at this stage demonstrated a profound flaccid and areflexic quadriparesis with no sensory disturbance and no involvement of the cranial nerves. Repeat lumbar puncture performed one week after admission showed a rise in CSF protein to $7.4 \mathrm{~g} / 1$ but was again otherwise normal, and all CSF cultures were negative. Serological tests for legionnaires' disease, chlamydia, syphilis and recent viral infection were negative, however, titre of both IgG and IgM antibodies to Mycoplasma sp. were significantly raised. On the basis of these findings, diagnosis of Guillain-Barré syndrome was made.

The patient's neurological status remained unchanged for a period of 8 weeks at which time he developed bilateral sixth nerve palsies and his conscious level deteriorated. A repeat CT scan revealed a large enhancing posterior fossa mass

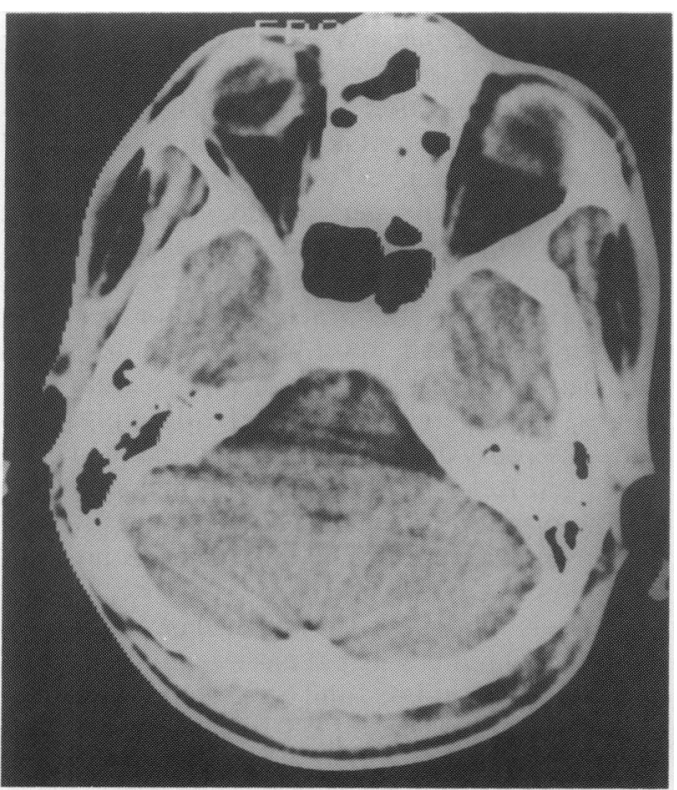

Figure 1 Post contrast CT scan through the pontomedullary junction showing normal appearances on admission. Note the prominent streak artifact obscuring posterior fossa detail. arising in the cervico-medullary junction and growing up into the obex (Figure 2). The fourth ventricle remained midline, and the third and lateral ventricles were dilated requiring ventriculo-peritoneal shunt insertion. CT cisternography at the time of shunt insertion and subsequent CT myelography demonstrated expansion of the medulla oblongata below the level of the third ventricle with almost complete block of the subarachnoid space at the level of the foramen magnum (Figure 3). The expansion of the medulla extended down into the spinal canal to approximately the level of the C1-C2 junction. Surgical exploration revealed a grey smooth gelatinous mass infiltrating the upper cervical cord in the region of $\mathrm{Cl}$ and extending superiorly into the obex to displace the cerebellar hemispheres laterally and the vermis cephalad. No attempt at resection was made although a superficial biopsy was obtained. Histological examination revealed a grade IV astrocytoma. The patient deteriorated rapidly after surgery and remained comatose until his death $\mathbf{8}$ days later.

\section{Discussion}

Guillain-Barré syndrome is one of a spectrum of inflammatory polyradiculoneuropathies which

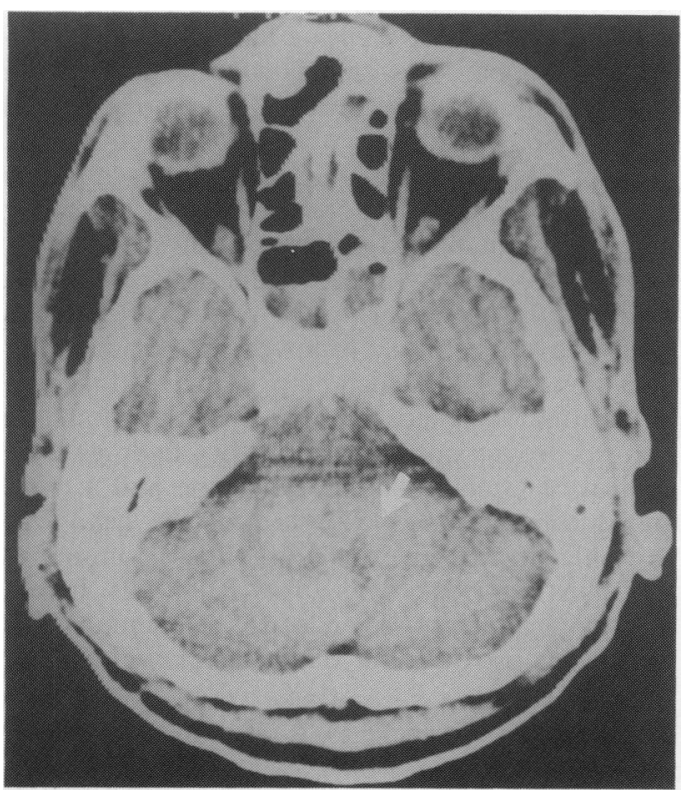

Figure 2 Post contrast CT scan 8 weeks after admission demonstrates an enhancing intrinsic mass lesion in the dorsal pons (arrow). 


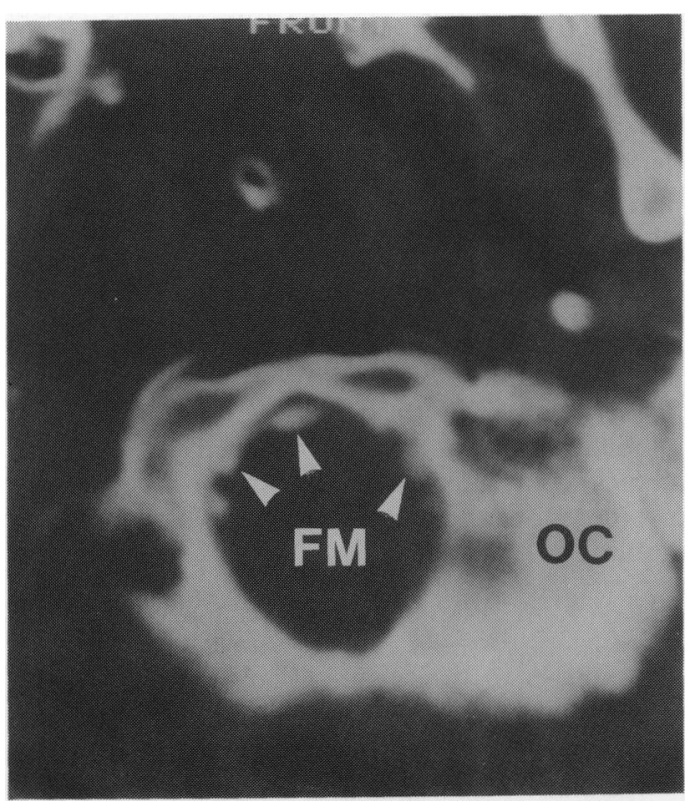

Figure 3 Post myelographic CT images through the level of the foramen magnum (FM) and occipital condyle (OC) demonstrate diffuse enlargement of the lower brain stem and upper spinal cord with an almost complete block to passage of contrast (arrows) at the level of the foramen magnum.

appears to result from an autoimmune response to infection. Positive serology indicating previous viral infection is seen in $40-60 \%$ of cases. Campylobacter jejuni and Mycoplasma pneumoniae are the commonest non-viral infection associated with Guillain-Barré syndrome and $M$. pneumonia appears to be responsible in approximately $5 \%$ of cases. $^{1,2}$ The clinical picture is of a rapidly progressive, symmetrical, areflexic flaccid paresis which develops over several days usually beginning in the lower limbs. Although muscle pains and paraesthesia are common, significant sensory loss is unusual. Cranial nerve involvement usually in the form of bilateral facial palsy is present in up to $50 \%$ of cases. ${ }^{2}$ One of the most distinctive findings is the presence of a markedly elevated CSF protein (greater than $0.6 \mathrm{~g} / \mathrm{l}$ ) which is mainly due to increased albumin content, although IgG, IgM and IgA levels are often slightly elevated..$^{1-4}$ This elevation of CSF protein is present in over $90 \%$ of cases in the first and second weeks of the illness, and may not peak for several weeks, often remaining elevated for months. The cell count in the CSF is seldom elevated above $0.01 \times 10^{9} / 1$ and immunoglobulin changes in the blood are minimal and nonspecific. ${ }^{1-3}$ Although there is no diagnostic test for Guillain-Barré syndrome, the clinical picture together with the typical CSF changes is usually diagnostic.

In the present case a malignant astrocytoma of the cervico-medullary junction replicated the clinical and CSF abnormalities leading to a mistaken diagnosis of Guillain-Barré syndrome. Although malignant gliomas of the cervicomedullary junction are extremely rare, it is of interest that Hankey ${ }^{2}$ mentions a similar case which was entered into a prospective study of Guillain-Barré syndrome and subsequently excluded when a glioma of the upper cervical cord was diagnosed.

In adults brainstem gliomas are rare, forming less than $5 \%$ of intracranial tumours of which less than $10 \%$ are malignant. ${ }^{5,6}$ Malignant brainstem gliomas are commonest in the pons, although up to $30 \%$ occur at the cervico-medullary junction. ${ }^{5-7}$ Clinical presentation is one of very rapid onset of progressive neurological impairment with extremely variable clinical signs depending on the site of origin of the tumour. ${ }^{6,7} \mathrm{CSF}$ abnormalities are invariably present and marked elevation of CSF protein is common. ${ }^{5,6}$

In the present case the tumour presumably arose in the ventral aspect of the upper cervical cord where invasion of the medial lemniscus and pyramidal tract caused paraesthesia and progressive motor deficit mimicking the clinical presentation of Guillain-Barré syndrome. ${ }^{8}$ It is important to realize that intrinsic tumours arising higher in the brainstem or at lower levels in the spinal cord would not cause dissociated motor weakness since motor and sensory pathways lie in close proximity. ${ }^{8}$

Imaging studies of the cervico-medullary junction are notoriously difficult and the normal initial CT scan was one of the major obstacles to the diagnosis of the present case. Brainstem gliomas exhibit an infiltrative growth pattern and are only rarely associated with oedema or enhancement so that CT reveals only subtle enlargement of the brainstem. ${ }^{5,9,10}$ This may be extremely difficult to appreciate, particularly at the cervico-medullary junction where streak artifact is inevitable. The introduction of magnetic resonance imaging (MRI) has improved imaging of the brainstem and spinal cord, and CT will miss up to $20 \%$ of brainstem gliomas demonstrated on MRI. ${ }^{5,7,9,10}$ Furthermore, MRI is not generally available and is associated with considerable difficulties in ventilated patients.

The most effective clinical test to distinguish between the polyradiculopathy of Guillain-Barré syndrome and motor paresis of central origin is the measurement of peripheral nerve conduction. In the present case, nerve conduction studies were not performed but would have been expected to be normal. In contrast Guillain-Barré is associated with slowing of motor conduction velocity and 
decreased amplitude of motor potentials, although these changes may not be seen in the early stages of the disease when inflammation is limited to the nerve roots.

Although CSF protein analysis was not performed in the present case, it is possible that it may have provided a diagnostic clue. The elevation of CSF protein in Guillain-Barré syndrome is almost entirely due to an increase in albumin content and is thought to represent passage of plasma contents through the damaged blood-brain barrier. ${ }^{3,4}$ The elevation of CSF protein associated with spinal cord and brainstem tumours can be similar in composition but may sometimes demonstrate elevation of one or more of the globulin components due to tumoral protein synthesis.

In conclusion an intrinsic tumour of the cervico-

\section{References}

1. Sutherland, J.M. Guillain-Barré syndrome. Med J Aust 1987, 146: 122-123.

2. Hankey, G.J. Guillain-Barré syndrome in Western Australia, 1980-1985. Med J Aust 1987, 146: 130-133.

3. Jensen, $K$. Cerebral fluid proteins in neurological diseases. Acta Neurol Scand 1978, 58: 75-87.

4. Weber, E.L. Electrophoretic analysis of cerebrospinal fluid proteins in patients with central nervous system mass lesions. J Neurosurg 1972, 36: 679-686.

5. Hueftle, M.G., Han, J.S., Kaufman, B. et al. MR imaging of brain stem gliomas. J Comp Assist Tomog 1985, 9: 263-267.

6. Cohen, A.R., Wisoff, J.H., Allen, J.C. et al. Malignant astrocytomas of the spinal cord. J Neurosurg 1989, 70: 50-54. medullary junction may mimic the clinical presentation and CSF findings classically associated with Guillain-Barré syndrome. The location of a tumour with these clinical features is most likely to be the cervico-medullary junction where CT is of least use. CSF protein analysis may provide a clue as to the underlying pathology but may also be in keeping with Guillain-Barré syndrome. Wherever possible we would recommend confirmation of the diagnosis of Guillain-Barré syndrome by measurement of peripheral nerve motor potentials. Preservation of motor potentials, elevation of CSF globulin level or any variation from the expected clinical course, as seen in the present case, should prompt more aggressive neuroradiological investigation.

7. Epstein, F.J. \& Farmer, J.P. Brain stem glioma growth patterns. $J$ Neurosurg 1993, 78: 408-412.

8. Walton, J.N. (ed.) Diseases of the Nervous System. Oxford University Press, Oxford, 1977, pp. 16-51.

9. Lee, B.C., Kneeland, J.B., Walker, R.W. et al. MR imaging of brain stem tumors. Am J Neurorad 1985, 6: 159-163.

10. Wilson, W.B., Kosmorsky, G.S., deMasters, B.K. et al. Diffusely infiltrating brainstem gliomas: A report of the diagnostic difficulties in 2 cases. Neurology 1990, 40: 1237-1241. 